\title{
Twelve-month outcomes of patients admitted to the acute general medical service at Groote Schuur Hospital
}

\author{
H Stuart-Clark, N Vorajee, S Zuma, L van Niekerk, V Burch, P Raubenheimer, J G Peter
}

Objectives. Hospitalisation for medical illness has ongoing impact on individuals, healthcare services and society beyond discharge. This study's objective was to determine the 12-month mortality and functional outcomes of patients admitted to the acute medical service at Groote Schuur Hospital (GSH).

Methods. Follow-up, using the hospital records system and provincial death registry, together with telephonic interviews or home visits, was attempted for 465 medical inpatients admitted to GSH between 14 September and 16 November 2009. Functional outcomes were assessed using the Katz activities of daily living (ADL) score and Barthel index (BI).

Outcome measures. The major study outcomes included: 12-month mortality (overall and unexpected), changes in functional status and pre- and post-admission employment rates.

Results. Inpatient mortality was 11\%. At 12-month follow-up, 35\% (145/415) were deceased and 30\% (125/415) could not be traced; $38 \%(55 / 145)$ of deaths were considered expected and unexpected mortality was associated with age $>40$ years $(p=0.02)$ and an admission urea $>7.0 \mathrm{mmol} / \mathrm{l}(p=0.004)$. Katz ADL deteriorated in $15 \%(21 / 143)$ of interviewed patients and was associated with age $>50$ years $(p=0.005) ; 23 \%(33 / 143)$ had improved Katz ADL associated with admission human immunodeficiency virus (HIV) $(p=0.01)$, tuberculosis (TB) infection $(p=0.05)$ and sepsis $(p=0.02)$. Employment rates declined from $41 \%(59 / 145)$ pre-admission to $18 \%(26 / 145)$ at 12 months $(p<0.001)$, with little increase in the number of persons receiving disability grants. Twenty per cent (29/145) of patients required hospital readmission and this was associated with ADL functional decline $(p=0.01)$.

Conclusions. There was a very high overall mortality of $42 \%$ in patients admitted to the general medical wards. Significant employment decline and readmission rates highlight the additional economic and societal burdens of hospitalisation due to medical illness in the survivors.

S Afr Med J 2012;102(6):549-553.
General medical illnesses, severe enough to necessitate hospitalisation, are known to have profound impacts on individuals, families, communities and healthcare services. Inpatient mortality and acute functional declines aside, hospitalised medical patients post discharge are known to have higher 12 -month mortality rates ${ }^{1}$ and be at increased risk for progressive functional declines. Families are burdened with long-term care-giving responsibilities and the financial implications of employment loss. In addition 'hospitalisation begets hospitalisation' and already overburdened health services face the increased utilisation of resources through hospital readmissions or outpatient visits. Furthermore, the high burden of illness in the setting of a country faced with the dual epidemics of human immunodeficiency virus (HIV) and chronic diseases of lifestyle places constant pressure on the optimal utilisation of acute hospital beds. Detailed short-, intermediate- and long-term follow-up and outcomes data are crucial to a better understanding of these impacts, and to inform patients, healthcare workers and policy makers.

Short-term outcomes, such as inpatient mortality and acute functional decline following hospitalisation, have been extensively studied and are well established for a number of medical illnesses in both developed and developing country settings. However, few

Department of Medicine, University of Cape Town

H Stuart-Clark, MB ChB

N Vorajee, $\mathrm{MB} C \mathrm{ChB}$

S Zuma, $\mathrm{MB}$ ChB

L van Niekerk, MB ChB, MSc Public Health (London)

V Burch, MB BCh, MMed, PhD, FCP (SA), FRCP (London)

P Raubenheimer, MB BCh, FCP (SA)

J G Peter, MB ChB, FCP (SA) data are available on the intermediate- and long-term outcomes of general medical hospital admissions, especially in developing country settings where documentation and follow-up are problematic. To our knowledge, and despite the fact that the majority of African hospitals provide generalist secondary-level medical services, there are no intermediate-term outcome studies of general medical cohorts from high HIV-prevalent developing countries. Studies from developed country settings are largely restricted to elderly or intensive care unit (ICU) cohorts ${ }^{1}$ with low HIV prevalence, or to specific medical conditions (e.g. ischaemic heart disease $\mathrm{e}^{2}$ or cerebrovascular disease $\mathrm{e}^{3}$ and their disease-specific outcomes). The South African healthcare context is unique. With intersecting burdens of communicable and non-communicable disease, we aimed to conduct a 12-month followup study of a cohort of hospitalised, medical inpatients admitted to a tertiary South African hospital.

\section{Methods}

\section{Study population}

Our study population consisted of 465 patients admitted to the acute general medical wards at Groote Schuur Hospital (GSH), Cape Town, between 14 September and 16 November 2009. During the 9-week study period all patients aged $>16$ years, referred from either the emergency unit or a district-level hospital to GSH, were eligible for inclusion and enrolled. Patients directly admitted to the ICU, those with acute coronary syndromes, patients admitted to palliative care beds (where death is anticipated within 48 hours of admission) and those admitted to the 'short-stay ward' (where patients are admitted with predominantly chronic disease and have an anticipated hospital stay $<48$ hours) were excluded. Verbal consent was obtained from all patients and the study received ethics approval from the University of Cape Town Human Research Ethics Committee. On admission, all baseline demographic and clinical information and pre-admission functionality data were collected. After discharge, folders underwent blinded review to document discharge diagnoses and identify patients 
Table 1. Demographic and basic clinical details for all study patients, and stratified by 12-month follow-up outcome

\begin{tabular}{|c|c|c|c|c|c|}
\hline $\begin{array}{l}\text { Patient demographics and } \\
\text { clinical characteristics }\end{array}$ & $\begin{array}{l}\text { All } \\
(N=415) \\
n(\%)\end{array}$ & $\begin{array}{l}\text { Deceased } \\
(N=145) \\
n(\%)\end{array}$ & $\begin{array}{l}\text { Interviewed } \\
(N=145) \\
n(\%)\end{array}$ & $\begin{array}{l}\text { Untraceable } \\
(N=125) \\
n(\%)\end{array}$ & $p$-value ${ }^{*}$ \\
\hline \multicolumn{6}{|l|}{ Age } \\
\hline Median (IQR) & $44(31-60)$ & $50(36-68)^{\dagger 1, \uparrow 2}$ & $39(29-56)^{\dagger 1}$ & $37(29-52)^{\dagger 2}$ & $<0.001^{\dagger 1},<0.001^{\dagger 2}$ \\
\hline Female & $224(54)$ & $74(51)$ & $84(58)$ & $69(56)$ & NS \\
\hline \multicolumn{6}{|l|}{ Racial group } \\
\hline Black & $216(46)$ & $51(35)^{\dagger 1}$ & $65(45)^{\dagger 2}$ & $75(60)^{\dagger 1, \uparrow 2}$ & $<0.001^{\dagger 1}, 0.01^{\star 2}$ \\
\hline White & $36(8)$ & $8(6)$ & $12(8)$ & $12(10)$ & NS \\
\hline Coloured & $204(44)$ & $83(58)$ & $63(43)$ & $37(30)$ & NS \\
\hline Other & $9(2)$ & $3(1)$ & $5(4)$ & $0(0)$ & NS \\
\hline \multicolumn{6}{|l|}{ Educational level } \\
\hline$<5$ years & $57(12)$ & $19(13)$ & $16(11)$ & $16(13)$ & NS \\
\hline $5-7$ years & $48(10)$ & $21(15)$ & $11(8)$ & $13(10)$ & NS \\
\hline$>7$ years & $262(56)$ & $74(51)$ & $98(68)$ & $77(62)$ & NS \\
\hline Unknown & $98(21)$ & $31(21)$ & $29(14)$ & $19(15)$ & NS \\
\hline HIV-positive & $146(31)$ & $39(27)$ & $42(29)$ & $50(40)$ & NS \\
\hline \multicolumn{6}{|l|}{ CD4, cells/ml $(n=96)$} \\
\hline Median (IQR) & $111(61-231)$ & $72(24-180)^{\dagger 1, \dagger 2}$ & $120(73-256)^{\dagger 1}$ & $153(65-302)^{\dagger 2}$ & $0.05^{\dagger 1}, 0.05^{\dagger 2}$ \\
\hline Pre-admission HAART & $56(38)$ & $15(10)$ & $17(12)$ & $20(16)$ & NS \\
\hline Fully independent pre-admission ADL & $298(72)$ & $97(67)^{\dagger 1}$ & $103(71)$ & $98(78)^{\dagger 1}$ & $0.04^{\dagger 1}$ \\
\hline Inpatient delirium & $74(16)$ & $28(20)^{\dagger 1}$ & $14(10)^{\dagger 1}$ & $17(14)$ & $0.02^{\dagger 1}$ \\
\hline \multicolumn{6}{|l|}{ MEWS } \\
\hline Median(IQR) & $2(1-4)$ & $2(1-4)$ & $2(1-4)$ & $2(1-4)$ & NS \\
\hline \multicolumn{6}{|l|}{ Urea } \\
\hline Median (IQR) & $6.6(4.4-12.3)$ & $7.9(4.9-16.8)^{\dagger 1, \dagger 2}$ & $6.0(4.1-10.4)^{\dagger 1}$ & $6.0(3.9-10.1)^{\dagger 2}$ & $0.002^{\dagger 1}, 0.002^{\dagger 2}$ \\
\hline Length of hospital stay & $6(4-11)$ & $6(3-10)$ & $6(3-10)$ & $7(4-12)$ & NS \\
\hline
\end{tabular}

in whom death might have been anticipated within 12 months post discharge. Standardised prognostic indicator guidelines were used to identify these patients. ${ }^{4,5}$

\section{Twelve-month follow-up}

All patients' contact details (identity numbers, telephone numbers and addresses) were obtained from the clinical records on the 'Clinicom' system (Clinicom is the provincial hospital computer record-keeping system used at GSH) and from the patients' folders, when otherwise unavailable. Additionally, Clinicom and/or the Western Cape provincial death registry was searched for details about patient mortality, date of death, hospital readmissions and/or outpatient clinic visits. Telephonic interviews were then attempted for all patients approximately 12 months following discharge from hospital. When patients were unable to be contacted by telephone, but had a valid address, a community healthcare worker undertook home visits. Patients unable to be contacted by any of the above methods were considered 'lost-to-follow-up'.

\section{Functional outcome assessments}

Functional outcome assessments were conducted at either telephonic interview or a home visit. Where possible, patients were interviewed, but if unavailable, the interview was conducted with a first-degree relative with adequate knowledge of patient status. A standardised questionnaire in the patient's/family member's first language was used. Data collected included a pre- and post-admission Katz

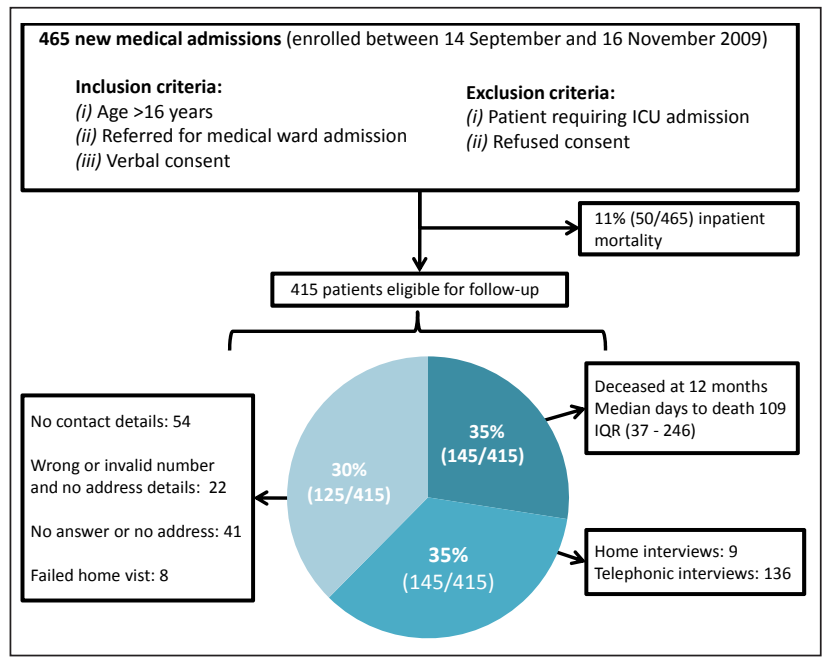

Fig. 1. Flow diagram indicating the 3 major 12-month follow-up outcomes.

activities of daily living (ADL) scale and Barthel index (BI), preand post-admission employment and disability grant status, and post-discharge readmissions, as well as outpatient, emergency room and private doctor visits. Both the BI and Katz ADL scales are standardised, validated indices used to assess patient functionality and independence. The BI is an ordinal scale considering 10 factors; independence in basic living activities gives a score out of 90; the Katz 
ADL score, although similar to the $\mathrm{BI}$, assesses $6 \mathrm{ADL}$ and categorises patients A - G, with category A equating to full independence. ${ }^{7}$

\section{Statistical analysis}

Simple descriptive statistics were employed and the 12-month deceased, untraceable and interviewed patient groups were compared using chi-squared and Kruskal-Wallis tests accordingly. Odds ratios (ORs) (95\% confidence interval (CI)) were calculated for predictors of 12-month mortality (and unexpected 12-month mortality) as well as for an improvement or deterioration in functional status using logistic regression analysis. For this analysis, 'untraceable' patients were considered to be alive since their names could not be found in the provincial death registry. A change of one category and 5 points was considered clinically significant for the Katz ADL scale and BI, respectively. In the univariate analysis for predictors of overall and unexpected 12-month mortality, age and HIV-positivity were treated as potential confounders when considered to be independently associated with both the predictor and mortality, e.g. age and cancer, HIV and tuberculosis (TB). STATA IC, version 10 (Stata Corp, Texas, USA) was used for all statistical analyses.

\section{Results}

During the 9-week study period, 465 patients referred for admission to the acute general medical wards were enrolled. Eleven per cent (55/465) of patients died during hospital admission, and consequently 12-month follow-up was attempted for 415 patients. Fig. 1 illustrates the 3 major follow-up outcome groups and details regarding each subgroup. Despite intensive efforts (including access to the provincial death registry), 30\% (125/415) of patients could not be traced at 12 months. There was no association between lower levels of education and 'lost-to-follow-up' category $(p=0.98)$. However, being fully independent for ADL pre-admission and being of African race were

Table 2. Univariate demographic and clinical associations of 12-month mortality

\begin{tabular}{|c|c|c|}
\hline Patient characteristics $^{\dagger}$ & Odds ratio $(95 \% \mathrm{CI})$ & $p$-value \\
\hline \multicolumn{3}{|c|}{ All 12-month mortality $(N=145)$} \\
\hline Age $>50$ years & $1.9(1.2-3.0)$ & 0.01 \\
\hline Urea $>7.0 \mathrm{mmol} / \mathrm{l}$ & $2.0(1.2-3.2)$ & 0.007 \\
\hline Inpatient delirium & $2.1(1.0-4.3)$ & 0.05 \\
\hline \multicolumn{3}{|c|}{ Primary admission diagnosis ${ }^{\ddagger}$} \\
\hline Cancer & $9.3(1.1-77.2)$ & 0.04 \\
\hline Respiratory disease $\mathrm{S}^{\S}$ & $8.5(1.0-72.0)$ & 0.05 \\
\hline TB & $1.2(0.5-2.7)$ & 0.66 \\
\hline \multicolumn{3}{|c|}{ Main underlying chronic diagnosis } \\
\hline HIV-positive & $1.5(0.9-2.7)$ & 0.15 \\
\hline \multicolumn{3}{|l|}{ If HIV-positive: } \\
\hline $\mathrm{CD} 4$ & $0.995(0.989-0.999)$ & 0.04 \\
\hline Cancer & $4.6(1.3-17.2)$ & 0.02 \\
\hline \multicolumn{3}{|c|}{ Unexpected 12-month mortality $(n=56)$} \\
\hline Age $>40$ years & $2.3(1.1-4.5)$ & 0.02 \\
\hline Urea $>7.0 \mathrm{mmol} / 1$ & $2.8(1.0-5.7)$ & 0.004 \\
\hline \multicolumn{3}{|c|}{$\begin{array}{l}\text { Of the } 145 \text { patients in the cohort that were deceased at } 12 \text { months, folder discharge data } \\
\text { were available for } 110 \text { patients only ( } 35 \text { folders missing). }\end{array}$} \\
\hline \multicolumn{3}{|c|}{$\begin{array}{l}\text { Age was treated as a potential confounder when evaluating all other variables. } \\
\text { "Primary admission diagnosis was considered the patients' reason for acute medical admission. } \\
\text { 'Respiratory admission diagnoses included asthma and chronic obstructive pulmonary } \\
\text { disease (COPD). } \\
\text { '3/145 deceased patients had no underlying chronic diagnosis. }\end{array}$} \\
\hline
\end{tabular}

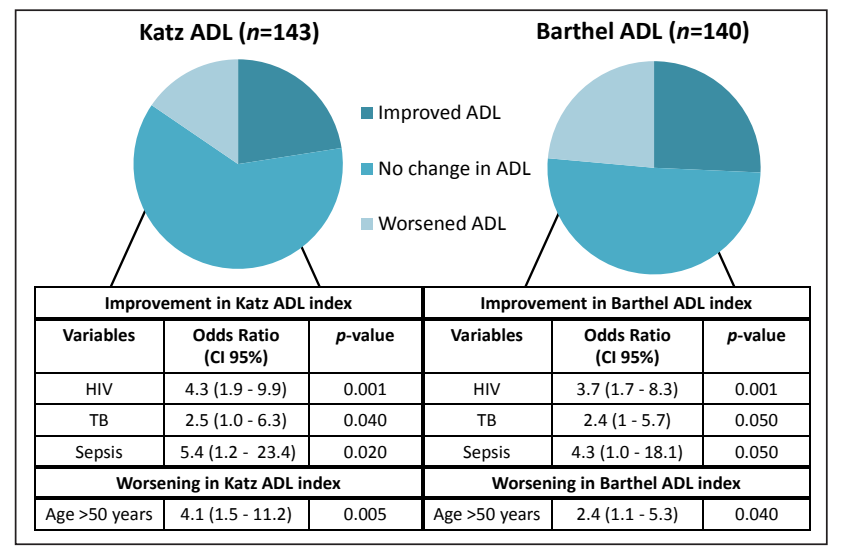

Fig. 2. Twelve-month follow-up changes in independent functioning as measured by the Katz and Bartel ADL indices and association with improvement or deterioration (only factors that were significantly associated with ADL improvement or worsening have been tabulated).TB and sepsis have been modelled with HIV as a potential confounder.

associated with lost-to-follow-up ( $p=0.002$ and $p=0.01$, respectively). Thirty-five per cent (145/415) of patients in the cohort were traced via telephonic interview or home visit.

Demographic and clinical details of all study patients, stratified by major outcome groups, are presented in Table 1. Overall, the median (interquartile range (IQR)) age was $44(31-50)$, and racial and educational demographics reflected known provincial statistics. Patients who were deceased at 12-month follow-up were significantly older than both those interviewed and lost-to-follow-up $(p<0.001)$. Thirty-one per cent $(146 / 415)$ were known to be HIV positive with no differences in prevalence between outcome groups. In HIV-infected patients, the median (IQR) CD4 cell count was $111(61-231)$.

Of the HIV-infected patients $38 \%(56 / 146)$ were receiving highly active antiretroviral therapy (HAART) pre-admission with no differences between groups. The majority of patients (72\%) admitted to the hospital were fully independent prior to admission, and median (IQR) length of hospital stay was $6(4-11)$ days. On admission, the median (IQR) modified early warning score (MEWS) was $2(1-4)$; notably, scores were not significantly higher in patients deceased at 12-month follow-up. Only $14 \%$ (68/465) of patients were categorised as having a high-risk MEWS $>5$. However, inpatient delirium occurred in $20 \%(28 / 145)$ of deceased patients compared with only $10 \%(14 / 145)$ of those available for interviews $(p=0.02)$.

Twelve-month mortality in the study cohort was 35\% (114/415). In the $80 \%(116 / 145)$ of patients where the date of death was known, the median (IQR) days-to-death was 109 (37 - 246) with 46\% (53/116) of deaths occurring in the first 3 months following hospital discharge; $38 \%(55 / 145)$ of 12 -month mortality was anticipated by the discharge clinician (with 23\% (34/145) of deceased patient folders not available for review). Combined inpatient and 12-month mortality was $42 \%$ (195/465). Table 2 illustrates demographic and clinical associations of 12-month mortality stratified by overall and unexpected 12-month mortality. Age $>50$ years, an admission urea $>7 \mathrm{mmol} / \mathrm{l}$ and inpatient delirium were associated with an increased likelihood of 12-month mortality ( $p=0.01, p=0.007$ and $p=0.05$, respectively). Additionally, a primary diagnosis of cancer and respiratory disease increased overall 12 -month mortality ( $p=0.04$ and $p=0.05$, respectively). The only demographic or clinical associations with unexpected 12 -month mortality were age $>40$ years and admission urea $>7.0 \mathrm{mmol} / \mathrm{l}$ $(p=0.02$ and $p=0.004)$. No specific primary admission diagnosis or chronic underlying medical condition was associated with a significant increase in unexpected 12-month mortality. In HIV- 
infected patients, 12-month mortality was $27 \%$ (39/145), with the combined inpatient and 12-month mortality being 28\% (54/195). HIV infection was not associated with an increased likelihood of 12-month mortality $(p=0.15)$.

Pre- and 12-month post-discharge functional ADL assessments using the Katz ADL scale and BI were completed for 143 and 140 patients, respectively. Fig. 2 illustrates the changes between preadmission and 12-month ADL functionality for the two indices and the important admission determinants of worse, or improved, ADL functionality. Results were similar for both indices (51\% and 62\% of patients' Katz ADL and BI scores, respectively, did not change from pre-admission levels); $23 \%$ and $26 \%$ of patients' Katz ADL and BI scores, respectively, showed improvement at 12 months post discharge. Whether the BI or Katz ADL index was used, improvement in ADL functioning was associated with HIV positivity (Katz ADL OR (95\% CI) 3.2 ( $1.3-7.7), p=0.01)$; the same proved true for an admission diagnosis of TB (Katz ADL OR (95\% CI) 2.5 (1.0 - 6.3), $p=0.04)$ or sepsis (Katz ADL OR (95\% CI) $5.4(1.2-23.4), p=0.02)$. In $15 \%$ and $23 \%$ of patients, Katz ADL and BI scores, respectively, had worsened at 12 months post discharge. A worsening ADL functionality, using either index, was associated only with an age $>50$ years (Katz ADL OR (95\% CI) 4.1 (1.5 - 11.2), $p=0.005)$.

At 12 -month follow-up only $18 \%$ (27/145) of interviewed patients were in active employment compared with 33\% (48/145) prior to hospitalisation $(p=0.005)$. A worsening Katz ADL score was associated with employment loss (OR ( $95 \% \mathrm{CI}) 3.0$ (1.1 - 8.7), $p=0.04$ ). Despite the loss of employment among interviewed patients, there was not a significant increase in the number of people accessing disability grants $(p=0.11)$. Of interviewed patients $19 \%(27 / 145)$ required readmission in the 12 months following discharge, with a median (range) of $1(1-6)$ hospitalisations per patient. Additionally, $47 \%(68 / 145), 5 \%(7 / 145)$ and $10 \%(15 / 145)$ of interviewed patients had accessed healthcare services in the form of outpatient consultations (range 4 - 10 occasions), emergency room visits $(1-2)$ and private general practitioner consultations ( 1 - 3), respectively. A worsening Katz ADL score was associated with hospital readmission (OR (95\% CI) 3.5 (1.3 - 9.2), $p=0.01$ ).

\section{Discussion}

This is the first descriptive study of the 12-month outcomes of a cohort of patients admitted to an acute general medical service in a South African hospital. It highlights serious impacts deriving from severe medical illness that necessitates admission to hospital and important future implications for both the individual and public health. The key study findings were: $(i)$ a very high 12-month mortality associated with known risk factors, but interestingly, not with HIV-infection; (ii) more than a third of 12-month mortality could be anticipated by clinicians at discharge, revealing the very high potential burden of patients on palliative care services, were they available, but who are accessing care as acute general medical admissions, and among whom (iii) approximately $20 \%$ required hospital readmission; (iv) an improvement in the ADL functioning of close to a quarter of patients interviewed at 12 months post discharge and the association of improvement with admission for chronic, but treatable, conditions such as HIV and TB; $(v)$ a $>80 \%$ reduction in employment rates associated with declining functionality; and ( $v i)$ the inability, despite modern record-keeping systems and household visits, to trace all patients.

While it is known that inpatient mortality in the general medical wards of GSH is approximately $10 \%$ (unpublished data), the 12-month mortality rates from acute general medical inpatient cohorts has not been previously established. The high 12 -month mortality of $35 \%$, and high combined overall cohort mortality rate of $42 \%$ (inpatient and outpatient 12-month deaths together), are comparable to those of follow-up studies of ICU cohorts in developed countries. ${ }^{1,8}$ This may be explained, in part, by the admission of a large number of patients with end-stage disease, who would fulfil criteria for referral to palliative care services. Unexpectedly, given that a third of patients were HIV-infected with a median CD4 cell count $<200$ cells $/ \mathrm{ml}$, and that less than half were receiving HAART pre-admission, HIV infection was found not to be associated with either an increased risk of overall or unexpected 12-month mortality. In fact, the inpatient mortality rate was lower than in a cohort of hospitalised HIV-infected patients with similar median CD4 counts in West Africa, ${ }^{9}$ and the 12-month mortality rate was only marginally higher than the 5 -year figures from a local primary care ARV-clinics study, ${ }^{10}$ reflecting the success of local infectious disease services and ARV-clinic treatment programmes. Moreover, an admission diagnosis of both HIV and TB infection was associated with improved functionality at 12 months in interviewed patients. These data suggest that patients deriving the greatest benefit from an acute hospital admission are those who are younger with acute deteriorations in chronic, yet treatable, conditions such as HIV and TB.

South Africa's inpatient medical services rival those in many developed countries, yet high lost-to-follow-up rates remain a major obstacle to ensuring high-quality long-term care. Patient migration and social and economic deprivation, together with inadequate record-keeping systems and personnel, mean that follow-up of patients with chronic and severe medical illness is a significant challenge. Lost-to-follow-up rates of between $5 \%$ and $15 \%$ are not uncommon in research and community clinic settings in South Africa. ${ }^{11,12}$ From a public health perspective, the need for strengthening hospital record-keeping, as well as the human resources to improve service delivery, is exposed.

Our study has a number of limitations. The large lost-to-follow-up number means that study conclusions regarding functional outcomes should be interpreted cautiously. Nonetheless, even if patients with expected mortality are removed, close to a third of cohort patients were deceased at 12 months. Moreover, important demographic and clinical characteristics (Table 1) did not differ significantly between the interviewed patients and those who were lost to followup. Therefore, it is unlikely that the addition of these patients to the functional outcome data would have significantly altered our conclusions. Accuracy of follow-up data collected using telephonic interviews, despite being conducted in patients' first language, is dependent on individual and/or family recall; this is an important study limitation, carrying the implication that hospital readmission rates and outpatient visits may be underestimates.

This study clearly identifies patients admitted to general medical wards as a very high-risk group for early mortality. A larger study is required to identify clear predictors of unexpected early mortality. Until such data are available, it may be prudent that patients discharged from a tertiary hospital be cared for as a high-risk group, arguably, with specialist follow-up to ensure optimal post-discharge treatment care. At present, the majority of patients admitted to GSH's general medical services are not afforded specialist follow-up after discharge, but are referred to their local community health centres. Whether more intensive follow-up (and the required resources that would be required) might reduce early mortality is a hypothesis that deserves to be tested. The requirement for improved palliative services for such patients is highlighted.

Author contributions. HSC and NV are equally contributing first authors. JGP and VB designed the study. HSC, NV, SZ, LV generated 
the data. HSC, NV, SZ, PR and JGP analysed the data. All authors were involved in the manuscript preparation.

\section{References}

1. Eddleston JM, White P, Guthrie E. Survival, morbidity, and quality of life after discharge from intensive care. Crit Care Med 2000;28(7):2293-2299.

Smith K, Ross D, Connolly E. Investigating six-month health outcomes of patients with angina discharged from a chest pain service. Eur J Cardiovasc Nurs 2002;1(4):253-264

3. Herlitz J, Karlson BW, Sjolin M, Lindquist J. Ten-year mortality for patients discharged after hospitalization for chest pain or other symptoms raising suspicion of acute myocardial infarction in relation to hospital discharge diagnosis. J Intern Med 2002;251(6):526-532.

4. Boyd K, Murray SA. Recognising and managing key transitions in end of life care. BMJ 2010;341:c4863.

5. Centre National Gold Standards Framework. The GSF Prognostic Indicator Guidance. England 2008.

www.goldstandardsframework.nhs.uk/.../CrawlerResourceServer.asp (accessed February 2012).

6. Mahoney FI, Barthel DW. Functional evaluation: The Barthel Index. Md State Med J 1965;14:61-65.
7. Katz S, Downs TD, Cash HR, Grotz RC. Progress in development of the index of ADL. Gerontologist 1970;10(1):20-30.

8. Broomhead LR, Brett SJ. Clinical review: intensive care follow-up - what has it told us? Crit Care 2002;6(5):411-417.

9. Saleri N, Capone S, Pietra V, et al. Outcome and predictive factors of mortality in hospitalized HIV patients in Burkina Faso. Infection 2009;37(2):142-147.

10. Boulle A, van Cutsem G, Hilderbrand K, et al. Seven-year experience of a primary care antiretroviral treatment programme in Khayelitsha, South Africa. AIDS 2010;24(4):563-572.

1. Wang B, Losina E, Stark R, et al. Loss to follow-up in a community clinic in South Africa - roles gender, pregnancy and CD4 count. S Afr Med J 2011;101(4):253-257.

12. Dheda K, Davids V, Lenders L, et al. Clinical utility of a commercial LAM-ELISA assay for TB diagnosis in HIV-infected patients using urine and sputum samples. PLoS One 2010;5(3):e9848.

Accepted 2 February 2012. 